\title{
SURFACES OF REVOLUTION IN THE THREE DIMENSIONAL PSEUDO-GALILEAN SPACE
}

\author{
DAE WON YOON \\ Gyeongsang National University, South Korea
}

\begin{abstract}
In the present paper, we study surfaces of revolution in the three dimensional pseudo-Galilean space $G_{3}^{1}$. Also, we characterize surfaces of revolution in $G_{3}^{1}$ in terms of the position vector field and Gauss map.
\end{abstract}

\section{INTRODUCTION}

Let $\mathbf{x}: M \longrightarrow \mathbb{E}^{m}$ be an isometric immersion of a connected $n$ dimensional manifold in the $m$-dimensional Euclidean space $\mathbb{E}^{m}$. Denote by $H$ and $\Delta$ the mean curvature and the Laplacian of $M$ with respect to the Riemannian metric on $M$ induced from that of $\mathbb{E}^{m}$, respectively. Takahashi ([16]) proved that the submanifolds in $\mathbb{E}^{m}$ satisfying $\Delta \mathbf{x}=\lambda \mathbf{x}$, that is, all coordinate functions are eigenfunctions of the Laplacian with the same eigenvalue $\lambda \in \mathbb{R}$ are either the minimal submanifolds of $\mathbb{E}^{m}$ or the minimal submanifolds of hypersphere $\mathbb{S}^{m-1}$ in $\mathbb{E}^{m}$.

As an extension of Takahashi theorem, Garay studied in [12] hypersurfaces in $\mathbb{E}^{m}$ whose coordinate functions are eigenfunctions of the Laplacian, but not necessarily associated to the same eigenvalue. He considered hypersurfaces in $\mathbb{E}^{m}$ satisfying the condition

$$
\Delta \mathbf{x}=A \mathbf{x},
$$

where $A \in \operatorname{Mat}(m, \mathbb{R})$ is an $m \times m$ - diagonal matrix, and proved that such hypersurfaces are minimal $(H=0)$ in $\mathbb{E}^{m}$ and open pieces of either round

2010 Mathematics Subject Classification. 53A35, 53B30.

Key words and phrases. Pseudo-Galilean space, surface of revolution, Gauss map.

This paper was supported by Basic Science Research Program through the National Research Foundation of Korea(NRF) funded by the Ministry of Education, Science and Technology(2012R1A1A2003994). 
hyperspheres or generalized right spherical cylinders. Related to this, Dillen, Pas and Verstraelen ([9]) investigated surfaces in $\mathbb{E}^{3}$ whose immersions satisfy the condition

$$
\Delta \mathbf{x}=A \mathbf{x}+B
$$

where $A \in \operatorname{Mat}(3, \mathbb{R})$ is a $3 \times 3$-real matrix and $B \in \mathbb{R}^{3}$. In other words, each coordinate function is of 1-type in the sense of Chen $([6])$. For the Lorentzian version of surfaces satisfying (1.2), Alías, Ferrández and Lucas ([1]) proved that the only such surfaces are minimal surfaces and open pieces of Lorentz circular cylinders, hyperbolic cylinders, Lorentz hyperbolic cylinders, hyperbolic spaces or pseudo-spheres.

The notion of an isometric immersion $\mathbf{x}$ is naturally extended to smooth functions on submanifolds of Euclidean space or pseudo-Euclidean space. The most natural one of them is the Gauss map of the submanifold. In particular, if the submanifold is a hypersurface, the Gauss map can be identified with the unit normal vector field to it. Dillen, Pas and Verstraelen ([10]) studied surfaces of revolution in the three dimensional Euclidean space $\mathbb{E}^{3}$ such that its Gauss map $G$ satisfies the condition

$$
\Delta G=A G, \quad A \in \operatorname{Mat}(3, \mathbb{R}) .
$$

Also, Baikoussis and Blair ([3]) investigated the ruled surfaces in $\mathbb{E}^{3}$ satisfying the condition (1.3), and Baikoussis and Verstraelen $([4,5])$ studied the helicoidal surfaces and the spiral surfaces in $\mathbb{E}^{3}$. Choi $([7,8])$ completely classified the surfaces of revolution and the ruled surfaces with non-null base curve satisfying the condition (1.3) in the three dimensional Minkowski space $\mathbb{E}_{1}^{3}$. Furthermore, Alías, Ferrández, Lucas and Meroño ([2]) studied the ruled surfaces with null ruling in $\mathbb{E}_{1}^{3}$, and Yoon ([17]) classified translation surfaces in $\mathbb{E}_{1}^{3}$.

The main purpose of this paper is to complete classification of surfaces of revolution in the three dimensional pseudo-Galilean space $G_{3}^{1}$ satisfying (1.1) and (1.3).

The study for some surfaces in Galilean or pseudo-Galilean space can find in $[11,13-15,18]$.

\section{Preliminaries}

The pseudo-Galilean space $G_{3}^{1}$ is a Cayley-Klein space with absolute figure consisting of the ordered triple $\{\omega, f, I\}$, where $\omega$ is the ideal (absolute) plane in the three dimensional real projective space $R P_{3}, f$ the line (the absolute line) in $\omega$ and $L$ the fixed hyperbolic involution of points of $f$.

Homogenous coordinates in $G_{3}^{1}$ are introduced in such a way that the absolute plane $\omega$ is given by $x_{0}=0$, the absolute line $f$ by $x_{0}=x_{1}=0$ and the hyperbolic involution $\eta$ by $\eta:\left(x_{0}: x_{1}: x_{2}: x_{3}\right) \rightarrow\left(0: 0: x_{3}: x_{2}\right)$. The last condition is equivalent to the requirement that the conic $x_{2}^{2}-x_{3}^{2}=0$ is the 
absolute conic. Metric relations are introduced with respect to the absolute figure. In affine coordinates defined by $\left(x_{0}: x_{1}: x_{2}: x_{3}\right)=(1: x: y: z)$, the distance between the points $P_{i}=\left(x_{i}, y_{i}, z_{i}\right)(i=1,2)$ is defined by (see [11])

$$
d\left(P_{1}, P_{2}\right)= \begin{cases}\left|x_{2}-x_{1}\right|, & \text { if } x_{1} \neq x_{2} \\ \sqrt{\left|\left(y_{2}-y_{1}\right)^{2}-\left(z_{2}-z_{1}\right)^{2}\right|}, & \text { if } x_{1}=x_{2}\end{cases}
$$

The group motions of $G_{3}^{1}$ is a six-parameter group given (in affine coordinates) by

$$
\begin{aligned}
& \bar{x}=a+x, \\
& \bar{y}=b+c x+y \cosh \varphi+z \sinh \varphi, \\
& \bar{z}=d+e x+y \sinh \varphi+z \cosh \varphi .
\end{aligned}
$$

Let $\mathbf{x}=\left(x_{1}, y_{1}, z_{1}\right)$ and $\mathbf{y}=\left(x_{2}, y_{2}, z_{2}\right)$ be vectors in $G_{3}^{1}$. A vector $\mathbf{x}$ is called isotropic if $x_{1}=0$, otherwise it is called non-isotropic. The pseudoGalilean scalar product of $\mathbf{x}$ and $\mathbf{y}$ is defined by

$$
\mathbf{x} \cdot \mathbf{y}= \begin{cases}x_{1} x_{2}, & \text { if } x_{1} \neq 0 \quad \text { or } \quad x_{2} \neq 0 \\ y_{1} y_{2}-z_{1} z_{2}, & \text { if } x_{1}=0 \text { and } x_{2}=0\end{cases}
$$

From this, the pseudo-Galilean norm of a vector $\mathbf{x}$ in $G_{3}^{1}$ is given by $\|\mathbf{x}\|=$ $\sqrt{|\mathbf{x} \cdot \mathbf{x}|}$ and all unit non-isotropic vectors are the form $\left(1, y_{1}, z_{1}\right)$. For isotropic vector $x_{1}=0$ holds. There are four types of isotropic vectors: spacelike $\left(y_{1}^{2}-z_{1}^{2}>0\right)$, timelike $\left(y_{1}^{2}-z_{1}^{2}<0\right)$ and the two types of lightlike $\left(y_{1}= \pm z_{1}\right)$ vectors. A non-lightlike isotropic vector is a unit vector if $y_{1}^{2}-z_{1}^{2}= \pm 1$.

The pseudo-Galilean cross product of $\mathbf{x}$ and $\mathbf{y}$ on $G_{3}^{1}$ is defined by

$$
\mathbf{x} \times \mathbf{y}=\left|\begin{array}{ccc}
0 & -e_{2} & e_{3} \\
x_{1} & y_{1} & z_{1} \\
x_{2} & y_{2} & z_{2}
\end{array}\right|
$$

where $e_{2}=(0,1,0)$ and $e_{3}=(0,0,1)$.

Consider a $C^{r}$-surface $M, r \geq 1$, in $G_{3}^{1}$ parametrized by

$$
\mathbf{x}(u, v)=\left(x\left(u_{1}, u_{2}\right), y\left(u_{1}, u_{2}\right), z\left(u_{1}, u_{2}\right)\right) .
$$

Let us denote $g_{i}=\frac{\partial x}{\partial u_{i}}, h_{i j}=\frac{\partial \tilde{\mathbf{x}}}{\partial u_{i}} \cdot \frac{\partial \tilde{\mathbf{x}}}{\partial u_{j}}(i, j=1,2)$, where $\sim$ stands for the projection of a vector on the pseudo-Euclidean $y z$-plane. Then, the matrix of the first fundamental form $d s^{2}$ of a surface $M$ in $G_{3}^{1}$ is given by

$$
d s^{2}=\left(\begin{array}{cc}
d s_{1}^{2} & 0 \\
0 & d s_{2}^{2}
\end{array}\right)
$$

where $d s_{1}^{2}=\left(g_{1} d u_{1}+g_{2} d u_{2}\right)^{2}$ and $d s_{2}^{2}=h_{11} d u_{1}^{2}+2 h_{12} d u_{1} d u_{2}+h_{22} d u_{2}^{2}$ (cf. [14]). In such case, we denote the components of $d s^{2}$ by $g_{i j}^{*}$. 
It is well known that in terms of local coordinates $\left\{u_{1}, u_{2}\right\}$ of $M$ the Laplacian operator $\Delta$ of the first fundamental form on $M$ is defined by

$$
\Delta=\frac{1}{\sqrt{|\mathfrak{g}|}} \sum_{i, j=1}^{2} \frac{\partial}{\partial u_{i}}\left(\sqrt{|\mathfrak{g}|} g^{* i j} \frac{\partial}{\partial u_{j}}\right),
$$

where $\mathfrak{g}=\operatorname{det}\left(g_{i j}^{*}\right)$ and $\left(g^{* i j}\right)=\left(g_{i j}^{*}\right)^{-1}$.

\section{Surfaces of ReVolution in $G_{3}^{1}$}

In the pseudo-Galilean space $G_{3}^{1}$, there are two types of rotations: pseudoEuclidean rotations given the normal form

$$
\begin{aligned}
& \bar{x}=x, \\
& \bar{y}=y \cosh t+z \sinh t, \\
& \bar{z}=y \sinh t+z \cosh t
\end{aligned}
$$

and isotropic rotations with the normal form

$$
\begin{aligned}
& \bar{x}=x+b t, \\
& \bar{y}=y+x t+b \frac{t^{2}}{2}, \\
& \bar{z}=z,
\end{aligned}
$$

where $t \in \mathbb{R}$ and $b=$ constant $>0$.

The trajectory of a single point under a pseudo-Euclidean rotation is a pseudo-Euclidean circle(i.e., a rectangular hyperbolic)

$$
x=\text { constant }, \quad y^{2}-z^{2}=r^{2}, \quad r \in \mathbb{R} .
$$

The invariant $r$ is the radius of the circle. Pseudo-Euclidean circles intersect the absolute line $f$ in the fixed points of the hyperbolic involution $\left(F_{1}, F_{2}\right)$. There are three kinds of pseudo-Euclidean circles: circles of real radius, of imaginary radius, and of radius zero. Circles of real radius are timelike curves (having timelike tangent vectors) and imaginary radius spacelike curves (having spacelike tangent vectors).

The trajectory of a point under isotropic rotation is an isotropic circle whose normal form is

$$
z=\text { constant }, \quad y=\frac{x^{2}}{2 b} .
$$

The invariant $b$ is the radius of the circle. The fixed line of the isotropic rotation (3.2) is the absolute line $f$.

First of all, we consider a nonisotropic curve $\alpha$ parametrized by

$$
\alpha(u)=(f(u), g(u), 0) \quad \text { or } \quad \alpha(u)=(f(u), 0, g(u))
$$


around the $x$-axis by pseudo-Euclidean rotation (3.1), where $g$ is a positive function and $f$ is a smooth function on an open interval $I$. Then the surface $M$ of revolution can be written as

$$
\mathbf{x}(u, v)=(f(u), g(u) \cosh v, g(u) \sinh v),
$$

$\mathrm{Or}$

$$
\mathbf{x}(u, v)=(f(u), g(u) \sinh v, g(u) \cosh v),
$$

for any $v \in \mathbb{R}$.

Next, we consider the isotropic rotations. By isotropic curve $\alpha(u)=$ $(0, f(u), g(u))$ about the $z$-axis by isotropic rotation (3.2), we obtain a surface

$$
\mathbf{x}(u, v)=\left(v, f(u)+\frac{v^{2}}{2 b}, g(u)\right),
$$

where $f$ and $g$ are smooth functions and $b \neq 0([14])$.

\section{Surfaces of Revolution Satisfying $\Delta \mathrm{x}=A \mathrm{x}$}

In this section, we classify surfaces of revolution in $G_{3}^{1}$ satisfying the equation

$$
\Delta \mathrm{x}=A \mathrm{x},
$$

where $A=\left(a_{i j}\right), i, j=1,2,3$.

First of all, let $M$ be a surface of revolution in $G_{3}^{1}$ defined by (3.3). Assume that the rotated curve $\alpha$ is parametrized by arc-length. Then it is rewritten as the form:

$$
\alpha(u)=(u, g(u), 0) .
$$

In this case, the parametrization of $M$ is given by

$$
\mathbf{x}(u, v)=(u, g(u) \cosh v, g(u) \sinh v),
$$

where $g$ is a positive function. From (2.3) we have

$$
g_{1}=1, g_{2}=0, h_{11}=h_{12}=0, h_{22}=-g^{2}(u),
$$

which imply the components of the first fundamental form $d s^{2}$ on $M$ are given by

$$
g_{11}^{*}=1, g_{12}^{*}=0, g_{22}^{*}=-g^{2}(u) .
$$

So, $M$ is a timelike surface. By a straightforward computation, the Laplacian operator $\Delta$ on $M$ with the help of (4.3) and (2.4) turns out to be

$$
\Delta=-\frac{g^{\prime}(u)}{g(u)} \frac{\partial}{\partial u}-\frac{\partial^{2}}{\partial u^{2}}+\frac{1}{g^{2}(u)} \frac{\partial^{2}}{\partial v^{2}} .
$$


Suppose that $M$ satisfies (4.1). Then from (4.2) and (4.4), we have

$$
\begin{aligned}
& a_{11} u+a_{12} g(u) \cosh v+a_{13} g(u) \sinh v=-\frac{g^{\prime}(u)}{g(u)} \\
& a_{21} u+a_{22} g(u) \cosh v+a_{23} g(u) \sinh v=\left(-\frac{g^{\prime}(u)^{2}}{g(u)}-g^{\prime \prime}(u)+\frac{1}{g(u)}\right) \cosh v \\
& a_{31} u+a_{32} g(u) \cosh v+a_{33} g(u) \sinh v=\left(-\frac{g^{\prime}(u)^{2}}{g(u)}-g^{\prime \prime}(u)+\frac{1}{g(u)}\right) \sinh v .
\end{aligned}
$$

Since the functions $\sinh v, \cosh v$ and the constant function are linearly independent, by (4.5) we get $a_{12}=a_{13}=a_{21}=a_{23}=a_{31}=a_{32}=0$ and $a_{22}=a_{33}=\mu$. Consequently the matrix $A$ satisfies

$$
A=\left(\begin{array}{lll}
\lambda & 0 & 0 \\
0 & \mu & 0 \\
0 & 0 & \mu
\end{array}\right)
$$

where $a_{11}=\lambda$ and (4.5) is rewritten as the following:

$$
\lambda u=-\frac{g^{\prime}(u)}{g(u)}, \quad \mu g(u)=-\frac{g^{\prime}(u)^{2}}{g(u)}-g^{\prime \prime}(u)+\frac{1}{g(u)} .
$$

Combining the first and the second equation of (4.6), we obtain

$$
g^{2}\left(2 \lambda^{2} u^{2}-\lambda+\mu\right)=1 .
$$

THEOREM 4.1. There is no harmonic surface of revolution given by (3.3) in the three dimensional pseudo-Galilean space $G_{3}^{1}$.

Proof. Suppose that $M$ satisfies $\Delta \mathbf{x}=0$. Then from (4.6) we obtain $g^{\prime}(u)=0$ and $\frac{1}{g(u)}=0$, it is impossible.

THEOREM 4.2. Let $M$ be a non-harmonic surface of revolution given by (3.3) in the three dimensional pseudo-Galilean space $G_{3}^{1}$. Then $M$ satisfies (4.1) if and only if it is an open part of a Lorentz hyperbolic cylinder.

Proof. If $2 \lambda^{2} u^{2}-\lambda+\mu=0$ in (4.7), then we get $\lambda=0$ and $\mu=0$, that is, $A=0$. It is contradiction because $M$ is a non-harmonic surface. So we assume $2 \lambda^{2} u^{2}-\lambda+\mu \neq 0$. Then from (4.7) we get

$$
g(u)= \pm \frac{1}{\sqrt{\left|2 \lambda^{2} u^{2}-\lambda+\mu\right|}} .
$$

If we substitute (4.8) in the first equation of (4.6), one find $\lambda=0$ and $g(u)=$ $\pm \frac{1}{\sqrt{|\mu|}}, \mu \neq 0$. It follows that $M$ is a Lorentz hyperbolic cylinder $y^{2}-z^{2}=\frac{1}{\mu}$ (see Figure 1). It can be easily shown that the converse assertion is also true. 


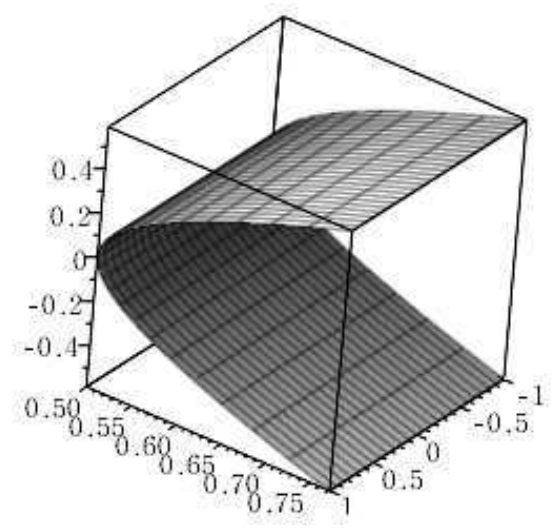

Figure 1. A Lorentz hyperbolic cylinder

Let $M$ be a surface of revolution given by (3.4) in the three dimensional pseudo-Galilean space $G_{3}^{1}$. Assume that a nonisotropic curve $\alpha$ is a unit speed curve. Then it is given by $\alpha(u)=(u, 0, g(u))$. In this case, the spacelike surface $M$ of revolution is parametrized by

$$
\mathbf{x}(u, v)=(u, g(u) \sinh v, g(u) \cosh v) .
$$

Suppose that the surface of revolution $M$ given by (4.9) satisfies (4.1). Then, by using similar method of Theorem 4.2, we have the following result:

THEOREM 4.3. Let $M$ be a non-harmonic surface of revolution given by (4.9) in the three dimensional pseudo-Galilean space $G_{3}^{1}$. Then $M$ satisfies (4.1) if and only if it is an open part of a hyperbolic cylinder $z^{2}-y^{2}=r^{2}$.

Last of all, let $M$ be a surface of revolution in $G_{3}^{1}$ generated by the rotated curve $\alpha(u)=(0, f(u), g(u))$. Assume that the curve $\alpha$ is parametrized by arclength, that is,

$$
f^{\prime}(u)^{2}-g^{\prime}(u)^{2}=-\epsilon(= \pm 1) .
$$

Then the parametrization of $M$ is given by

$$
\mathbf{x}(u, v)=\left(v, f(u)+\frac{v^{2}}{2 b}, g(u)\right),
$$

where $f$ and $g$ are smooth functions and $b \neq 0$. From (2.3) we have

$$
g_{1}=0, g_{2}=1, h_{11}=-\epsilon, h_{12}=h_{22}=0,
$$

which imply the components of the first fundamental form $d s^{2}$ on $M$ are given by

$$
g_{11}^{*}=1, g_{12}^{*}=0, g_{22}^{*}=-\epsilon .
$$


By (4.11) and (2.4) the Laplacian operator $\Delta$ of $M$ can be expressed as

$$
\Delta=\epsilon \frac{\partial^{2}}{\partial u^{2}}+\epsilon \frac{\partial^{2}}{\partial v^{2}}
$$

Suppose that $M$ satisfies (4.1). Then from (4.10) and (4.12), we have

$$
\begin{aligned}
& a_{11} v+a_{12}\left(f(u)+\frac{v^{2}}{2 b}\right)+a_{13} g(u)=0, \\
& a_{21} v+a_{22}\left(f(u)+\frac{v^{2}}{2 b}\right)+a_{23} g(u)=\epsilon f^{\prime \prime}(u)+\frac{\epsilon}{b}, \\
& a_{31} v+a_{32}\left(f(u)+\frac{v^{2}}{2 b}\right)+a_{33} g(u)=\epsilon g^{\prime \prime}(u) .
\end{aligned}
$$

It imply we can easily find $a_{11}=a_{12}=a_{13}=a_{21}=a_{22}=a_{31}=a_{32}=0$. In this case, (4.13) is rewritten as the following:

$$
\lambda g(u)=\epsilon f^{\prime \prime}(u)+\frac{\epsilon}{b}, \quad \mu g(u)=\epsilon g^{\prime \prime}(u),
$$

where $\lambda=a_{23}$ and $\mu=a_{33}$. The general solution of the second equation in (4.14) is given by

$$
g(u)=a_{1} u+a_{2}
$$

if $-\epsilon \mu=0$, where $a_{1}, a_{2}$ are constants,

$$
g(u)=b_{1} \cos \left(k u+b_{2}\right)
$$

if $-\epsilon \mu=k^{2}$, where $b_{1}, b_{2}$ and $k$ are constants, or

$$
g(u)=c_{1} \cosh \left(k u+c_{2}\right)
$$

if $\epsilon \mu=k^{2}$, where $c_{1}, c_{2}$ are constants. Thus from (4.15), (4.16), (4.17) and the first equation in (4.14) the function $f(u)$ is given by

or

$$
\begin{gathered}
f(u)=\frac{1}{6} \epsilon \lambda a_{1} u^{3}+\frac{1}{2}\left(\epsilon \lambda a_{2}-\frac{1}{b}\right) u^{2}+a_{3} u+a_{4}, \\
f(u)=-\frac{\epsilon b_{1} \lambda}{k^{2}} \cos \left(k u+b_{2}\right)-\frac{1}{2 b} u^{2}+b_{3} u+b_{4},
\end{gathered}
$$

$$
f(u)=\frac{\epsilon c_{1} \lambda}{k^{2}} \cosh \left(k u+c_{2}\right)-\frac{1}{2 b} u^{2}+c_{3} u+c_{4},
$$

where $a_{i}, b_{i}, c_{i}(i=1,2,3,4)$ and $k$ are constants.

Consequently, the following theorem holds.

THEOREM 4.4. A surface of revolution generated by the isotropic curve in the three dimensional pseudo-Galilean space $G_{3}^{1}$ satisfies the equation $\Delta \mathbf{x}=$ $A \mathbf{x}, A \in \operatorname{Mat}(3, \mathbb{R})$ if and only if the surface can be parametrized as

$$
\mathbf{x}(u, v)=\left(v, f(u)+\frac{v^{2}}{2 b}, g(u)\right),
$$


where

(i) either $f(u)=\frac{1}{6} \epsilon \lambda a_{1} u^{3}+\frac{1}{2}\left(\epsilon \lambda a_{2}-\frac{1}{b}\right) u^{2}+a_{3} u+a_{4}$ and $g(u)=a_{1} u+a_{2}$,

(ii) $f(u)=-\frac{\epsilon b_{1} \lambda}{k^{2}} \cos \left(k u+b_{2}\right)-\frac{1}{2 b} u^{2}+b_{3} u+b_{4}$ and $g(u)=b_{1} \cos \left(k u+b_{2}\right)$,

(iii) or $f(u)=\frac{\epsilon c_{1} \lambda}{k^{2}} \cosh \left(k u+c_{2}\right)-\frac{1}{2 b} u^{2}+c_{3} u+c_{4}$ and $g(u)=c_{1} \cosh \left(k u+c_{2}\right)$ with $a_{i}, b_{i}, c_{i}, k \in \mathbb{R}(i=1,2,3,4)$.

REMARK 4.5. For specific functions $f(u)$ and $g(u)$ appropriate intervals of $u$ and $v$ in (i) and (ii), we have the graphs shown in Figure 2 and Figure 3 .

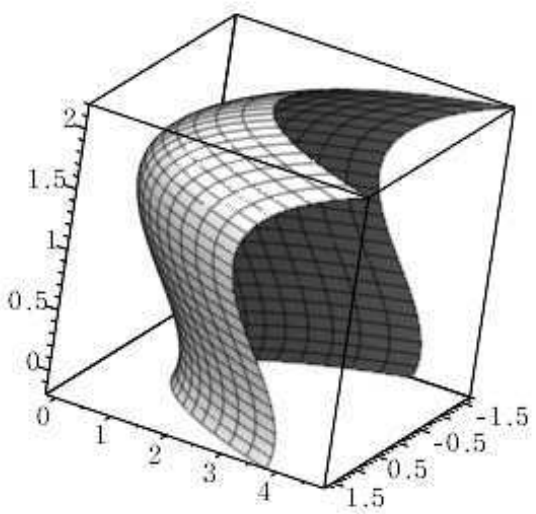

Figure 2. A timelike surface with $f(u)=u^{3}+\frac{3}{2} u^{2}-u$ and $g(u)=u+1$

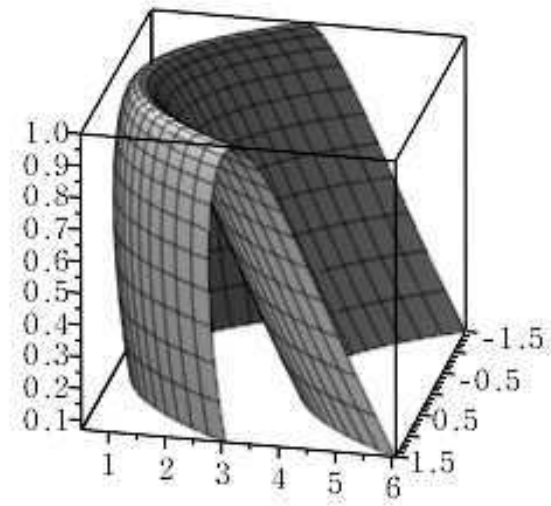

FIgURE 3. A spacelike surface with $f(u)=\cos (u)+u^{2}+u$ and $g(u)=\cos (u)$ 


\section{Surfaces of Revolution Satisfying $\Delta G=A G$}

In this section, we classify surfaces of revolution in $G_{3}^{1}$ satisfying the following equation

$$
\Delta G=A G
$$

where $A=\left(a_{i j}\right), i, j=1,2,3$.

THEOREM 5.1. Let $M$ be a surface of revolution given by (4.2) in the three dimensional pseudo-Galilean space $G_{3}^{1}$. Then $M$ satisfies (5.1) if and only if it is an open part of a Lorentz hyperbolic cylinder.

Proof. Let $M$ be a surface of revolution generated by a unit speed nonisotropic curve in $G_{3}^{1}$. Then $M$ is parametrized by

$$
\mathbf{x}(u, v)=(u, g(u) \cosh v, g(u) \sinh v),
$$

where $g$ is a positive function. From the natural frame $\left\{\mathbf{x}_{u}, \mathbf{x}_{v}\right\}$ the Gauss map $G$ of $M$ is obtained by

$$
G=\left(\frac{1}{\left\|\mathbf{x}_{u} \times \mathbf{x}_{v}\right\|}\right) \mathbf{x}_{u} \times \mathbf{x}_{v}=(0, \cosh v, \sinh v) .
$$

Suppose that $M$ satisfies (5.1). Then from (4.4) and (5.3) we get the following system of differential equations:

$$
\begin{aligned}
& a_{12} \cosh v+a_{13} \sinh v=0, \\
& a_{22} \cosh v+a_{23} \sinh v=\frac{1}{g^{2}(u)} \cosh v, \\
& a_{32} \cosh v+a_{33} \sinh v=\frac{1}{g^{2}(u)} \sinh v .
\end{aligned}
$$

In order to prove the theorem we have to solve the above system of ordinary differential equations. From (5.4) we easily deduce that

$$
a_{12}=a_{13}=a_{23}=a_{32}=0, a_{22}=a_{33}
$$

and

$$
\frac{1}{g^{2}(u)}=a_{22} \text {. }
$$

From this $g(u)$ is a constant function. Consequently, $M$ is an open part of a Lorentz hyperbolic cylinder. It can be easily shown that the converse assertion is also true.

TheOREM 5.2. Let $M$ be a surface of revolution given by (4.9) in the three dimensional pseudo-Galilean space $G_{3}^{1}$. Then $M$ satisfies (5.1) if and only if it is an open part of a hyperbolic cylinder. 
Let $M$ be a surface of revolution generated by a unit speed isotropic curve in $G_{3}^{1}$. Then $M$ is parametrized by

$$
\mathbf{x}(u, v)=\left(v, f(u)+\frac{v^{2}}{2 b}, g(u)\right) .
$$

From this the Gauss map $G$ of $M$ is given by

$$
G=\left(0,-g^{\prime}(u),-f^{\prime}(u)\right) .
$$

Suppose that $M$ satisfies (5.1). Then from (4.12) and (5.7) we get the following system of differential equations:

$$
\begin{aligned}
& a_{12} g^{\prime}(u)+a_{13} f^{\prime}(u)=0, \\
& a_{22} g^{\prime}(u)+a_{23} f^{\prime}(u)=\epsilon g^{\prime \prime \prime}(u), \\
& a_{32} g^{\prime}(u)+a_{33} f^{\prime}(u)=\epsilon f^{\prime \prime \prime}(u) .
\end{aligned}
$$

If $f^{\prime}(u)=0$, then $g(u)= \pm u+c_{1}$ and $\epsilon=1$. Also, we find $a_{12}=a_{22}=$ $a_{32}=0$ and the matrix $A$ satisfies

$$
A=\left(\begin{array}{lll}
a_{11} & 0 & a_{13} \\
a_{21} & 0 & a_{23} \\
a_{31} & 0 & a_{33}
\end{array}\right) .
$$

Thus, $M$ is parametrized by

$$
\mathbf{x}(u, v)=\left(v, c_{2}+\frac{v^{2}}{2 b}, \pm u+c_{1}\right),
$$

where $c_{1}, c_{2}$ are constants, and $M$ is timelike parabolic cylinder.

If $g^{\prime}(u)=0$, then $f(u)= \pm u+d_{1}$ and $\epsilon=-1$. From (5.8), (5.9) and (5.10) we get $a_{13}=a_{23}=a_{33}=0$ and the matrix $A$ satisfies

$$
A=\left(\begin{array}{lll}
a_{11} & a_{12} & 0 \\
a_{21} & a_{22} & 0 \\
a_{31} & a_{32} & 0
\end{array}\right) .
$$

Thus, $M$ is parametrized by

$$
\mathbf{x}(u, v)=\left(v, \pm u+\frac{v^{2}}{2 b}+d_{1}, d_{2}\right)
$$

where $d_{1}, d_{2}$ are constants, and $M$ is spacelike plane.

We suppose that $f^{\prime}(u) \neq 0$ and $g^{\prime}(u) \neq 0$. If $a_{12} \neq 0$, then from (5.8) we get

$$
g^{\prime}(u)=-\frac{a_{13}}{a_{12}} f^{\prime}(u)
$$

Substituting $g^{\prime \prime \prime}(u)$ in (5.9) and comparing it and (5.10), we have

$$
a_{22}=a_{32}, a_{23}=a_{33}, a_{13}=-a_{12} .
$$


It follows that we can obtain the following ODEs

$$
\begin{aligned}
f^{\prime}(u) & =g^{\prime}(u), \\
f^{\prime \prime \prime}(u) & =\epsilon\left(a_{32}+a_{33}\right) f^{\prime}(u)
\end{aligned}
$$

and the relation

$$
a_{32}+a_{33}=a_{22}+a_{23} .
$$

Thus, we have the general solution of (5.13) as follows

$$
f(u)=\frac{a_{1}}{k} \sin \left(k u+a_{2}\right)+a_{3}
$$

if $\epsilon\left(a_{32}+a_{33}\right)=-k^{2}$,

$$
f(u)=\frac{b_{1}}{k} \sinh \left(k u+b_{2}\right)+b_{3}
$$

if $\epsilon\left(a_{32}+a_{33}\right)=k^{2}$ or

$$
f(u)=\frac{1}{2} c_{1} u^{2}+c_{2} u+c_{3}
$$

if $\epsilon\left(a_{32}+a_{33}\right)=0$, where $a_{i}, b_{i}, c_{i}(i=1,2,3)$ are constants.

THEOREM 5.3. A surface of revolution generated by the isotropic curve in the three dimensional pseudo-Galilean space $G_{3}^{1}$ satisfies the equation $\Delta G=$ $A G, A \in \operatorname{Mat}(3, \mathbb{R})$ if and only if the surface can be parametrized as

$$
x(u, v)=\left(v, f(u)+\frac{v^{2}}{2 b}, g(u)\right),
$$

where

(i) either $f(u)=d_{1}$ and $g(u)= \pm u+d_{2}$,

(ii) $f(u)= \pm u+d_{3}$ and $g(u)=d_{4}$,

(iii) $f(u)=\frac{1}{2} c_{1} u^{2}+c_{2} u+c_{3}$ and $g(u)=\frac{1}{2} c_{1} u^{2}+c_{2} u+c_{3}$,

(iv $f(u)=\frac{a_{1}}{k} \sin \left(k u+a_{2}\right)+a_{3}$ and $g(u)=\frac{a_{1}}{k} \sin \left(k u+a_{2}\right)+a_{3}$

(v) or $f(u)=\frac{b_{1}}{k} \sinh \left(k u+b_{2}\right)+b_{3}$ and $g(u)=\frac{b_{1}}{k} \sinh \left(k u+b_{2}\right)+b_{3}$.

REMARK 5.4. For specific functions $f(u)$ and $g(u)$ appropriate intervals of $u$ and $v$ in (iii), we have the graph shown in Figure 4.

Theorem 5.5. (ClassifiCATION). Let $M$ be a surface of revolution given by (4.2) in the three dimensional pseudo-Galilean space $G_{3}^{1}$. Then, for some matrix $A \in \operatorname{Mat}(3, \mathbb{R})$ the following are equivalent:

(1) $\Delta \mathbf{x}=A \mathbf{x}$

(2) $\Delta G=A G$.

(3) $M$ is an open part of a Lorentz hyperbolic cylinder. 


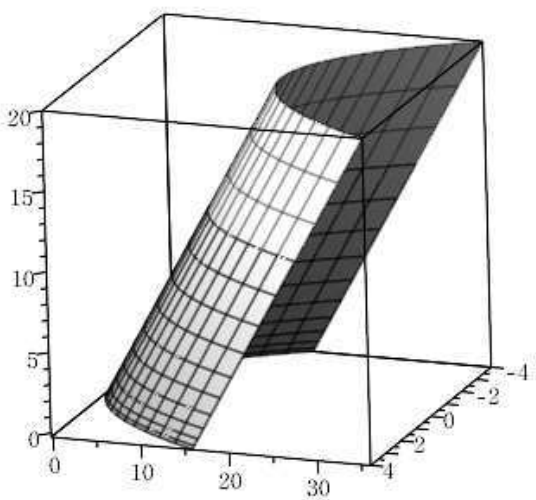

FIGURE 4. A surface of revolution with $f(u)=u^{2}+u+1$ and $g(u)=u^{2}+u+1$.

TheOrem 5.6. (ClassifiCATION). Let $M$ be a surface of revolution given by (4.9) in the three dimensional pseudo-Galilean space $G_{3}^{1}$. Then, for some matrix $A \in \operatorname{Mat}(3, \mathbb{R})$ the following are equivalent:

(1) $\Delta \mathbf{x}=A \mathbf{x}$.

(2) $\Delta G=A G$.

(3) $M$ is an open part of a hyperbolic cylinder.

Acknowledgements.

The author wishes to express their sincere thanks to the referee for making several useful comments.

\section{REFERENCES}

[1] L. J. Alías, A. Ferrández and P. Lucas, Surfaces in the 3-dimensional LorentzMinkowski space satisfying $\Delta x=A x+B$, Pacific J. Math. 156 (1992), 201-208.

[2] L. J. Alías, A. Ferrández, P. Lucas and M. A. Meroño, On the Gauss map of Bscrolls, Tsukuba J. Math. 22 (1998), 371-377.

[3] C. Baikoussis and D. E. Blair, On the Gauss map of ruled surfaces, Glasgow Math. J. 34 (1992), 355-359.

[4] C. Baikoussis and L. Verstraelen, On the Gauss map of helicoidal surfaces, Rend. Sem. Math. Messina Ser. II 2(16) (1993), 31-42.

[5] C. Baikoussis and L. Verstraelen, The Chen-type of the spiral surfaces, Results Math. 28 (1995), 214-223.

[6] B.- Y. Chen, A report on submanifold of finite type, Soochow J. Math. 22 (1996), 117-337.

[7] S. M. Choi, On the Gauss map of surfaces of revolution in a 3-dimensional Minkowski space, Tsukuba J. Math. 19 (1995), 351-367.

[8] S. M. Choi, On the Gauss map of ruled surfaces in a 3-dimensional Minkowski space, Tsukuba J. Math. 19 (1995), 285-304.

[9] F. Dillen, J. Pas and L. Vertraelen, On surfaces of finite type in Euclidean 3-space, Kodai Math. J. 13 (1990), 10-21. 
[10] F. Dillen, J. Pas and L. Vertraelen, On the Gauss map of surfaces of revolution, Bull. Inst. Math. Acad. Sinica 18 (1990), 239-246.

[11] B. Divjak and Ž. Milin Šipuš, Some special surface in the pseudo-Galilean space, Acta Math. Hungar. 118 (2008), 209-226.

[12] O. J. Garay, An extension of Takahashi's theorem, Geom. Dedicata 34 (1990), 105112.

[13] Ž. Milin Šipuš, Ruled Weingarten surfaces in the Galilean space, Period. Math. Hungar. 56 (2008), 213-225.

[14] Ž. Milin Šipuš and B. Divjak, Surfaces of constant curvature in the pseudo-Galilean space, Int. J. Math. Math. Sci. 2012, 1-28.

[15] Ž. Milin Šipuš and B. Divjak, Translation surface in the Galilean space, Glas. Mat. Ser. III 46(66) (2011), 455-469.

[16] T. Takahashi, Minimal immersions of Riemannian manifolds, J. Math. Soc. Japan 18 (1966), 380-385.

[17] D. W. Yoon, On the Gauss map of translation surfaces in Minkowski 3-space, Taiwanese J. Math. 6 (2002), 389-398.

[18] D. W. Yoon, Some classification of translation surfaces in Galilean 3-space, Int. J. Math. Anal. (Ruse) 6 (2012), 1355-1361.

D. W. Yoon

Department of Mathematics Education and RINS

Gyeongsang National University

Jinju 660-701

South Korea

E-mail: dwyoon@gnu.ac.kr

Received: 30.10 .2012$.

Revised: 5.3.2013. \& 14.3.2013. 